\title{
糖尿病家族史对妅娠糖尿病孕妇血糖、血脂及 姃娠结局的影响
}

周雨梅 ${ }^{1}$, 谢 妮 $^{2}$, 张丽霞 ${ }^{1}$, 陈丹青 ${ }^{1}$

1. 浙江大学医学院附属妇产科医院产科, 浙江杭州 310006

2. 浙江省杭州市采荷街道卫生服务中心, 浙江 杭州 310018

[摘要] 目的:探究糖尿病家族史对妊娠糖尿病(GDM)孕妇血糖、血脂以及妊娠 结局的影响。方法: 通过 1:4 匹配回顾性分析 2019年1-12月在浙江大学医学院附 属妇产科医院产科分娩的 GDM 孕妇 1265 例, 其中有糖尿病家族史组 253 例, 无糖尿病 家族史组 1012例。两组间血脂、血糖水平及妊娠结局 (大于胎龄儿、小于胎龄儿、 巨大儿、剖宫产、子痛前期、早产、产后出血、胎儿客迫) 差异采用 $t$ 检验和 $\chi^{2}$ 检 验, 糖尿病家族史与妊娠结局相关性分析采用二元Logistic 回归分析。结果: 有糖尿病 家族史组孕晚期高密度脂蛋白水平低于无糖尿病家族史组 $(P<0.05)$, 空腹血糖、口 服葡萄糖耐量试验 $($ OGTT $) 1 \mathrm{~h}$ 血糖、OGTT $2 \mathrm{~h}$ 血糖及糖化血红蛋白水平较无糖尿病 家族史组更高 $($ 均 $P<0.01)$ 。糖尿病家族史是GDM 孕妇子痛前期的独立危险因素 $(O R=3.27,95 \% C I: 1.39 \sim 7.68)$ 。结论: 有糖尿病家族史的 GDM 孕妇高密度脂蛋白水 平更低、血糖水平更高。糖尿病家族史是 GDM 孕妇发生子痛前期的独立危险因素。

[ 关键词 ] 妊娠糖尿病; 家族史; 血脂; 血糖; 子㾁前期; 危险因素

[中图分类号 ] R714.256 [ 文献标志码 ］A

\section{Impact of family history of diabetes on blood glucose, lipid} levels and perinatal outcomes in pregnant women with gestational diabetes mellitus

ZHOU Yumei ${ }^{1}$, XIE Ni ${ }^{2}$, ZHANG Lixia ${ }^{1}$, CHEN Danqing ${ }^{1}$ (1. Department of Obstetrics, Women's Hospital, Zhejiang University School of Medicine, Hangzhou 310006, China; 2. Caihe Community Health Service Center, Hangzhou 310018, China)

Corresponding author: CHEN Danqing, E-mail: chendq@zju.edu.cn, https://orcid.org/ 0000-0002-0201-7215

[ Abstract ] Objective: To investigate the impact of family history of diabetes (FHD) on blood glucose, lipid levels and perinatal outcomes in pregnant women with gestational

\section{收稿日期: 2021-04-29接受日期:2021-06-07}

基金项目:国家自然科学基金(81873839);浙江省重点研发计划(2018C03010)

第一作者:周雨梅,住院医师,主要从事产科学研究;E-mail:zym1780@zju.edu.cn;https ://orcid.org/0000-0002-3237-0677 通信作者:陈丹青,主任医师,教授,博士生导师,主要从事产科学研究;E-mail : chendq@zju.edu.cn; https ://orcid.org/00000002-0201-7215 
diabetes mellitus (GDM). Methods: A total of 1265 GDM women who gave childbirth in Women's Hospital, Zhejiang University School of Medicine during January to December 2019 were enrolled in the study, including 253 women with FHD and 1012 women without FHD. The $t$-test or $\chi^{2}$ test were used to compare the blood lipid, blood glucose levels and perinatal outcomes including large for gestational age infant, small for gestational age infant, macrosomia, cesarean delivery, preeclampsia, preterm labor, postpartum hemorrhage, fetal distress. The correlation between FHD and perinatal outcomes were estimated by Logistic regression analysis. Results: The high density lipoprotein level at third-trimester was significantly lower in GDM women with FHD $(P<0.05)$; and the women with FHD also had higher fasting blood glucose oral glucose tolerance test (OGTT)1 h, OGTT $2 \mathrm{~h}$ and glycosylated hemoglobin level (all $P<0.01$ ). In GDM women, FHD was an independent risk factor for preeclampsia $(O R=3.27,95 \% C I$ : 1.39-7.68). Conclusions: GDM women with FHD have lower high density lipoprotein and higher glucose levels. FHD is an independent risk factor for preeclampsia in GDM women.

[ Key words ] Gestational diabetes mellitus; Family history; Blood lipid; Blood glucose; Preeclampsia; Risk factor

[J Zhejiang Univ (Med Sci), 2021, 50(3): 329-334.]

[缩略语] 妊娠糖尿病 (gestational diabetes mellitus, GDM); 身体质量指数(body mass index, BMI); 口服葡萄糖耐量试验 (oral glucose tolerance test, OGTT); 甘油三酯(triglyceride, TG); 总胆固醇 (total cholesterol, TC); 高密度脂蛋白(high density lipoprotein, HDL); 低密度脂蛋白 (low density lipoprotein,LDL); 大于胎龄儿(large for gestational age infant,LGA); 小于胎龄儿 (small for gestational age infant, SGA); 比值比 (odds ratio, OR); 可信区间 (credibility interval,CI)

GDM 是指妊娠期首次发生或发现的糖尿 病 $^{[1]}$, 是常见的产科并发症, 与一系列不良围产结 局有关 ${ }^{[2]}$ 。目前, GDM 在全球范围内的发病率为 $1 \% \sim 30 \%{ }^{[3]}$, 在中国 GDM 的发病率为 $14.8 \%{ }^{[4]}$, 呈逐 年上升趋势。GDM 的危险因素包括糖尿病家族史、 前次妊娠GDM史、巨大儿分娩史、妊娠期高血压、高 龄及 BMI大于 $25.0 \mathrm{~kg} / \mathrm{m}^{2[5-6]}$, 其中糖尿病家族史是 GDM 的独立危险因素。糖尿病家族史与脂肪细胞 肥大、心血管疾病和亚临床动脉籿样硬化相关 ${ }^{[7-8]}$, 意味着糖尿病家族史可能参与糖脂代谢, 是 GDM 及 其并发症的协同因素。本研究通过回顾性分析有 糖尿病家族史孕妇 GDM 高发的原因及可能的阻断 方式,为临床改善GDM 孕妇的妊娠结局提供思路。

\section{1 对象与方法}

\section{1 对 象}

纳人 2019 年 $1-12$ 月在浙江大学医学院附属
妇产科医院产科门诊进行孕中期首次产检并于本 院分娩的孕妇。纳人标准 : (1)单胎; (2)孕中期 (24 28 周)行75 g OGTT 并诊断为 GDM ; 3分娩孕 周至少 28 周。排除标准:(1)孕前合并心脏病、高血 压疾病、肝功能不全、肾功能不全等严重脏器疾 病; (2)孕前合并糖尿病、甲状腺疾病等代谢性疾 病;3家族史不详。

参照国际糖尿病与妊娠协会研究组的 GDM 诊断标准:空腹血糖至少 $5.1 \mathrm{mmol} / \mathrm{L}, \mathrm{OGTT} 1 \mathrm{~h}$ 血 糖至少 $10.0 \mathrm{mmol} / \mathrm{L}$, OGTT $2 \mathrm{~h}$ 血糖至少 $8.5 \mathrm{mmol} / \mathrm{L}$, 符合其中任意一项。

根据年龄、孕次、产次、孕期增重和 BMI进行 1：4 匹配, 最终纳人 1265 例 GDM 孕妇, 其中有糖 尿病家族史 253 例, 无糖尿病家族史 1012 例。所 有研究对象均签署知情同意书, 研究经浙江大学 医学院附属妇产科医院伦理委员会审批 [(2019) 伦审科第 $(054)$ 号]。 


\section{2 观察指标}

观察指标包括年龄、孕次、孕前 BMI、孕期增 重、血糖水平、孕中期(孕24 28周)血脂水平、孕晚 期 (孕 32 36 周) 血脂水平及妊娠结局。糖尿病家 族史指一级亲属 (父母、子女及兄弟姐妹) 中有 2 型 糖尿病病史。观察指标及糖尿病家族史信息均从 病案信息中获得, 其中糖尿病家族史经由电话随 访确认。血脂水平包括 $\mathrm{TG} 、 \mathrm{TC} 、 \mathrm{HDL}$ 和 $\mathrm{LDL}$ 。妊 娠结局包括 LGA、SGA、巨大儿、剖宫产、子㾁前 期、早产、产后出血和胎儿莬迫。

\section{3 统计学方法}

采用SPSS 26.0软件进行统计学分析, 计量资 料采用均数 \pm 标准差 $(\bar{x} \pm s)$ 表示, 组间比较采用 $t$ 检验; 计数资料用例数和百分比 $[n(\%)]$ 表示, 组 间比较采用 $\chi^{2}$ 检验; 糖尿病家族史与妊娠结局的相 关性分析采用二元 Logistic 回归分析, 计算 $O R$ 值 和 $95 \% C I$ 值。以 $P<0.05$ 为差异有统计学意义。

\section{2 结 果}

2.1 有无糖尿病家族史 GDM 孕妇一般情况比较 两组 GDM 孕妇之间年龄、孕次、产次、孕前 B M I 孕期增重差异均无统计学意义 (均 $P>$ $0.05)$, 见表 1 , 提示两组间具有可比性,避免了年 龄、孕产次、孕前 BMI及孕期增重对分析结果的 影响。

2.2 有无糖尿病家族史 GDM 孕妇孕期血脂水平 比较

两组 GDM 孕妇孕中期血脂水平差异无统计 学意义,但有糖尿病家族史组孕晚期 HDL 水平低 于无糖尿病家族史组 $(P<0.05)$, 见表 2 。结果表 明, 在同样的干预措施下, 有糖尿病家族史组血脂 控制效果较差, 提示有糖尿病家族史的 GDM 孕妇 需要更严格的干预措施。
2.3 有无糖尿病家族史 GDM 孕妇孕期血糖水平 比较

有糖尿病家族史组空腹血糖、OGTT $1 \mathrm{~h}$ 血糖、 OGTT $2 \mathrm{~h}$ 血糖和糖化血红蛋白水平均高于无糖尿 病家族史组, OGTT 异常项数为二项或三项的患者 较多,空腹血糖和服糖后血糖均异常的比例更高 $($ 均 $P<0.01)$, 见表 3 。结果提示, 有糖尿病家族史 组糖耐量受损更严重。

2.4 有无糖尿病家族史 GDM 孕妇发生妊娠并发 症的多因素分析

在矫正了年龄、孕产次、孕前 BMI、孕期增重 之后, 通过Logistic 分析发现糖尿病家族史与发生 子痌前期存在正相关 $(O R=3.27,95 \% C I$ : 1.39 7. $68, P<0.01)$, 与其他妊娠并发症的发生无 相关性, 见表 4 。结果提示, 糖尿病家族史是 GDM 孕妇发生子痫前期的独立危险因素。

\section{3 讨 论}

随着科技进步、社会发展以及人们生活方式 的改变,妊娠期间被诊断为 GDM 的孕妇逐年增 多。妊娠糖尿病与一系列不良围产结局有关,如子 㾁前期、剖宫产、羊水过多、巨大儿、新生儿低血糖、 新生儿呼吸宕迫等, 同时也会增加GDM 孕妇及其 子代出现糖尿病、心血管疾病和肥胖的风险 ${ }^{[9-11] 。}$ 目前已有研究证实糖尿病家族史是GDM 的独立危 险因素 ${ }^{[12]}$ 。本研究通过回顾性分析有糖尿病家族 史孕妇 GDM 高发的原因及可能的阻断方式,以期 改善有糖尿病家族史GDM 孕妇的围产结局。

正常妊娠期间,为了满足胎儿生长发育的需 求,母体内脂质代谢发生变化。在妊娠晚期,胎儿 生长需求增加,脂肪库加速分解,胰岛素拮抗合并 胰岛素分泌相对不足导致脂蛋白脂肪酶活性下 降,TG 降解能力减弱, 导致 TG 水平较高, 而 HDL

表 1 有无糖尿病家族史妊娠糖尿病孕妇一般情况比较

Table 1 Clinical characteristics of gestational diabetes mellitus patients with and without family history of diabetes

\begin{tabular}{|c|c|c|c|c|c|c|c|c|}
\hline \multirow{2}{*}{ 组 别 } & \multirow{2}{*}{$n$} & \multirow{2}{*}{ 年龄(岁) } & \multirow{2}{*}{ 孕 次 } & \multicolumn{2}{|c|}{ 产 次 } & \multirow{2}{*}{$\begin{array}{l}\text { 孕前BMI } \\
\left(\mathrm{kg} / \mathrm{m}^{2}\right)\end{array}$} & \multirow{2}{*}{$\begin{array}{c}\text { 孕期增重 } \\
(\mathrm{kg})\end{array}$} & \multirow{2}{*}{$\begin{array}{c}\text { 分婏孕周 } \\
\text { (周) }\end{array}$} \\
\hline & & & & 初产妇 & 经产妇 & & & \\
\hline 有糖尿病家族史组 & 253 & $30.9 \pm 3.7$ & $2.0 \pm 1.2$ & $176(69.6)$ & $77(30.4)$ & $21.6 \pm 3.0$ & $13.5 \pm 3.9$ & $38.79 \pm 1.52$ \\
\hline 无糖尿病家族史组 & 1012 & $30.5 \pm 3.5$ & $1.9 \pm 1.1$ & $735(72.6)$ & $277(27.4)$ & $21.3 \pm 2.7$ & $13.8 \pm 4.1$ & $38.82 \pm 1.45$ \\
\hline$t / \chi^{2}$ 值 & - & -1.649 & -0.507 & \multicolumn{2}{|c|}{0.942} & -1.647 & 0.937 & 0.317 \\
\hline$P$ 值 & - & $>0.05$ & $>0.05$ & \multicolumn{2}{|c|}{$>0.05$} & $>0.05$ & $>0.05$ & $>0.05$ \\
\hline
\end{tabular}

“—”:无相关数据. BMI:身体质量指数. 
表 2 有无糖尿病家族史妊娠糖尿病孕妇孕期血脂水平比较

Table 2 Serum lipid levels of gestational diabetes mellitus patients with and without family history of diabetes

$(\bar{x} \pm s, \mathrm{mmol} / \mathrm{L})$

\begin{tabular}{|c|c|c|c|c|c|c|c|c|c|}
\hline \multirow[b]{2}{*}{ 组 别 } & \multirow[b]{2}{*}{$n$} & \multicolumn{4}{|c|}{ 孕中期 } & \multicolumn{4}{|c|}{ 孕晚期 } \\
\hline & & 总胆固醇 & 甘油三酯 & $\begin{array}{l}\text { 高密度 } \\
\text { 脂蛋白 }\end{array}$ & $\begin{array}{l}\text { 低密度 } \\
\text { 脂蛋白 }\end{array}$ & 总胆固醇 & 甘油三酯 & $\begin{array}{l}\text { 高密度 } \\
\text { 脂蛋白 }\end{array}$ & $\begin{array}{l}\text { 低密度 } \\
\text { 脂蛋白 }\end{array}$ \\
\hline 有糖尿病家族史组 & 253 & $6.14 \pm 1.04$ & $2.43 \pm 1.04$ & $2.33 \pm 0.55$ & $3.34 \pm 0.84$ & $6.50 \pm 1.33$ & $4.01 \pm 2.23$ & $1.98 \pm 0.49$ & $3.45 \pm 1.01$ \\
\hline 无糖尿病家族史组 & 1012 & $6.18 \pm 1.10$ & $2.32 \pm 0.84$ & $2.34 \pm 0.51$ & $3.40 \pm 0.91$ & $6.59 \pm 1.39$ & $3.77 \pm 1.99$ & $2.07 \pm 0.58$ & $3.54 \pm 1.05$ \\
\hline$t$ 值 & - & 0.412 & -1.685 & 0.321 & 0.854 & 0.967 & -1.640 & 2. 209 & 1. 264 \\
\hline$P$ 值 & - & $>0.05$ & $>0.05$ & $>0.05$ & $>0.05$ & $>0.05$ & $>0.05$ & $<0.05$ & $>0.05$ \\
\hline
\end{tabular}

“一” :无相关数据.

表 3 有无糖尿病家族史妊娠糖尿病孕妇孕期血糖水平比较

Table 3 Serum glucose levels of gestational diabetes mellitus patients with and without family history of diabetes

$[\bar{x} \pm s$ 或 $n(\%)]$

\begin{tabular}{|c|c|c|c|c|c|c|c|c|c|c|c|}
\hline \multirow[b]{2}{*}{ 组 别 } & \multirow[b]{2}{*}{$n$} & \multicolumn{4}{|c|}{ 血糖水平 $(\mathrm{mmol} / \mathrm{L})$} & \multicolumn{3}{|c|}{ OGTT异常项数 } & \multicolumn{3}{|c|}{ 血糖异常类型 } \\
\hline & & 空 腹 & OGTT $1 \mathrm{~h}$ & OGTT $2 \mathrm{~h}$ & $\begin{array}{l}\text { 糖化血红 } \\
\text { 蛋白 }\end{array}$ & $\begin{array}{l}\text { 一项 } \\
\text { 异常 }\end{array}$ & $\begin{array}{l}\text { 二项 } \\
\text { 异常 }\end{array}$ & $\begin{array}{l}\text { 三项 } \\
\text { 异常 }\end{array}$ & $\begin{array}{c}\text { 空腹血糖 } \\
\text { 异常 }\end{array}$ & $\begin{array}{c}\text { 服糖后血 } \\
\text { 糖异常 }\end{array}$ & $\begin{array}{c}\text { 前两者均 } \\
\text { 异常 }\end{array}$ \\
\hline $\begin{array}{c}\text { 有糖尿病家 } \\
\text { 族史组 }\end{array}$ & 253 & $4.9 \pm 0.6$ & $10.4 \pm 1.6$ & $8.9 \pm 1.4$ & $5.2 \pm 0.6$ & $\begin{array}{c}114 \\
(45.1)\end{array}$ & $\begin{array}{c}99 \\
(39.1)\end{array}$ & $\begin{array}{c}40 \\
(15.8)\end{array}$ & $\begin{array}{c}30 \\
(11.9)\end{array}$ & $\begin{array}{c}162 \\
(64.0)\end{array}$ & $\begin{array}{c}61 \\
(24.1)\end{array}$ \\
\hline $\begin{array}{c}\text { 无糖尿病家 } \\
\text { 族史组 }\end{array}$ & 1012 & $4.8 \pm 0.6$ & $9.9 \pm 1.4$ & $8.3 \pm 1.4$ & $5.1 \pm 0.3$ & $\begin{array}{c}692 \\
(68.4)\end{array}$ & $\begin{array}{c}265 \\
(26.2)\end{array}$ & $\begin{array}{c}55 \\
(5.4)\end{array}$ & $\begin{array}{c}165 \\
(16.3)\end{array}$ & $\begin{array}{c}730 \\
(72.1)\end{array}$ & $\begin{array}{c}117 \\
(11.6)\end{array}$ \\
\hline$t / \chi^{2}$ 值 & - & -2.697 & -4.743 & -5.933 & -3.680 & & 58.075 & & & 27.134 & \\
\hline$P$ 值 & - & $<0.01$ & $<0.01$ & $<0.01$ & $<0.01$ & & $<0.01$ & & & $<0.01$ & \\
\hline
\end{tabular}

“一” : 无相关数据. OGTT: 口服葡萄糖耐量试验.

表 4 有无糖尿病家族史妊娠糖尿病孕妇妊娠结局比较

Table 4 Pregnancy outcomes of gestational diabetes mellitus patients with and without family history of diabetes

\begin{tabular}{|c|c|c|c|c|c|c|c|c|}
\hline 组 别 & LGA & SGA & 巨大儿 & 子㾁前期 & 剖宫产 & 早 产 & 胎儿若迫 & 产后出血. \\
\hline 有糖尿病家族史组 & $63(24.9)$ & $6(2.4)$ & $27(10.7)$ & $11(4.3)$ & $128(50.6)$ & $17(6.7)$ & $15(5.9)$ & $3(1.2)$ \\
\hline 无糖尿病家族史组 & $201(19.9)$ & $28(2.8)$ & $88(8.7)$ & $15(1.5)$ & $447(44.2)$ & $51(5.0)$ & $64(6.3)$ & $12(1.2)$ \\
\hline OR值 & 1. 34 & 0.88 & 1.25 & 3.27 & 1.21 & 1. 28 & 0.93 & 0.86 \\
\hline $95 \% C I$ & $0.96 \sim 1.89$ & $0.36 \sim 2.16$ & $0.78 \sim 2.01$ & 1. 39 7. 68 & $0.91 \sim 1.62$ & $0.72 \sim 2.27$ & $0.52 \sim 1.67$ & $0.23 \sim 3.28$ \\
\hline$P$ 值 & $>0.05$ & $>0.05$ & $>0.05$ & $<0.01$ & $>0.05$ & $>0.05$ & $>0.05$ & $>0.05$ \\
\hline
\end{tabular}

$O R$ 值经过年龄、孕产次、孕前身体质量指数和孕期增重矫正. LGA : 大于胎龄儿; SGA : 小于胎龄儿.

水平较低 ${ }^{[13]}$ 。有研究发现, 糖尿病家族史与低 HDL 密切相关 ${ }^{[14-15]}$, 且有糖尿病家族史的 GDM 孕 妇 TG 水平较高 ${ }^{[16-17]}$ 。本文资料显示, 有糖尿病家 族史 GDM 孕妇与无糖尿病家族史 GDM 孕妇妊娠 中期的血脂水平差异无统计学意义,但有糖尿病 家族史组妊娠晚期的 HDL 水平低于无糖尿病家族 史组。有糖尿病家族史组 $\mathrm{TG}$ 水平也较无糖尿病 家族史组略增高, 但差异无统计学意义, 可能与我 院通过营养门诊和糖尿病一日门诊对糖尿病患者 进行及时篮查和积极干预有关。由于有糖尿病家
族史组 HDL 较低, 其在相同的干预措施下血脂控 制效果较差, 建议有糖尿病家族史的 GDM 患者除 了接受医学营养治疗外,还需要更严格的饮食控 制甚至口服降血脂药物。

本文资料显示,有糖尿病家族史组 OGTT 血 糖和糖化血红蛋白水平显著高于无糖尿病家族史 组, 服糖后血糖受损的比例更高, 且 OGTT 指标异 常数量明显高于无糖尿病家族史组, 可能与有糖 尿病家族史的孕妇糖尿病相关易感基因的表达导 致胰岛素分泌减少有关 ${ }^{[18-20]}$ 。迄今, 已有许多证 
据表明 GDM 与遗传学之间的关系。流行病学研 究表明,约 $50 \%$ 的 GDM 孕妇在孕后 10 年出现 2 型 糖尿病 ${ }^{[21-22]}$, 提示 GDM 与 2 型糖尿病有一些相同 的遗传风险位点。随着全基因组关联分析的出 现, CDKAL1、CDKN2A/2B、FTO、GCKR、HHEX、 IGF2BP2、KCNJ11、PPARG、SLC30A8和TCF7L2基 因突变已被证实与 2 型糖尿病相关 ${ }^{[23-26]}$ 。在此基 础上, 后续研究证实 $T C F 7 L 2 、 C D K A L 1 、 T C F 2$ 、 FTO、CDKN $2 A / 2 B 、 H H E X 、 I G F 2 B P 2$ 及 $S C L 30 A 8$ 基 因突变与 GDM 显著相关, 其中 CDKAL1、CDKN2A/ $2 B 、 H H E X$ 突变与胰岛素分泌减少有关 ${ }^{[27-28]}$ 。综 上, 有糖尿病家族史人群有更多 2 型糖尿病易感基 因的表达,这些易感基因突变可能导致胰岛素分 泌功能受损, 组织胰岛素受体敏感性下降, 胰岛素 拮抗合并胰岛素分泌相对不足,最终表现为糖耐 量受损。因此, 对于有糖尿病家族史的 GDM 患 者, 除医学营养和运动干预外, 还可能需要监测餐 后血糖, 甚至需要胰岛素和口服降糖药等进一步 干预。

一项队列研究表明,糖尿病家族史与子㾁前 期以及妊娠高血压相关 ${ }^{[29]}$, 本研究得出了相似的 结论, 但本研究仅发现糖尿病家族史是子戍前期 的独立危险因素。此外,已有研究发现妊娠期间 轻度糖耐量异常的孕妇中, 糖尿病家族史是 LGA 的预测指标 ${ }^{[30-31]}$ 。本文资料中, 有糖尿病家族史 组 GDM 孕妇巨大儿和剖宫产的风险较无糖尿病 家族史组增加, 这可能是因为糖尿病家族史与 GDM 孕妇胎儿餐后脑部活动有关, 继而影响胎儿 生长发育 ${ }^{[32]}$ 。根据这些结果, 推测糖尿病家族史 通过在 GDM 中诱导糖脂代谢异常从而导致多种 妊娠并发症, 但确切结论有待进一步研究证实。 因此, 对于有糖尿病家族史的 GDM 孕妇, 预防子 㾁前期是必要的。

综上所述,有糖尿病家族史的 GDM 孕妇 HDL 较低, 血糖水平较高, 糖尿病家族史是 GDM 孕妇 子㾁前期的独立危险因素。因此, 有糖尿病家族 史的 GDM 孕妇需要进行更严格的血脂和血糖控 制以及预防子㾁前期发生。

\section{利益冲突 所有作者均声明不存在利益冲突}

\section{参考文献}

[1] METZGER B E, GABBE S G, PERSSON B, et al.
International association of diabetes and pregnancy study groups recommendations on the diagnosis and classification of hyperglycemia in pregnancy $[\mathrm{J}]$. Diabetes Care, 2010, 33(3): 676-682.

[2] DAMM P, HOUSHMAND-OEREGAARD A, KELSTRUP L, et al. Gestational diabetes mellitus and long-term consequences for mother and offspring: a view from Denmark [J]. Diabetologia, 2016, 59(7): 1396-1399.

[3] MCINTYRE H D, CATALANO P, ZHANG C, et al. Gestational diabetes mellitus $[\mathrm{J}]$. Nat Rev Dis Primers, 2019, 5(1): 47.

[4] GAO C, SUN X, LU L, et al. Prevalence of gestational diabetes mellitus in mainland China: a systematic review and meta-analysis $[\mathbf{J}]$. J Diabetes Investig, 2019, 10(1): 154-162.

[5] LEE K W, CHING S M, RAMACHANDRAN V, et al. Prevalence and risk factors of gestational diabetes mellitus in Asia: a systematic review and meta-analysis $[\mathrm{J}]$. BMC Pregnancy Childbirth, 2018, 18(1): 494.

[6] AYDIN H, ÇELIK Ö, YAZICI D, et al. Prevalence and predictors of gestational diabetes mellitus: a nationwide multicentre prospective study $[\mathrm{J}]$. Diabet Med, 2019, 36(2): 221-227.

[7] DAHLMAN I, RYDEN M, ARNER P. Family history of diabetes is associated with enhanced adipose lipolysis: Evidence for the implication of epigenetic factors $[\mathrm{J}]$. Diabetes Metab, 2018, 44(2): 155-159.

[8] VORNANEN M, KONTTINEN H, KÄÄRIÄINEN H, et al. Family history and perceived risk of diabetes, cardiovascular disease, cancer, and depression $[\mathrm{J}]$. Prev Med, 2016, 90: 177-183.

[9] JENSEN D M, KORSHOLM L, OVESEN P, et al. Adverse pregnancy outcome in women with mild glucose intolerance: is there a clinically meaningful threshold value for glucose? $[J]$. Acta Obstet Gynecol Scand, 2008, 87(1): 59-62.

[10] FERRARA A, WEISS N S, HEDDERSON M M, et al. Pregnancy plasma glucose levels exceeding the American Diabetes Association thresholds, but below the National Diabetes Data Group thresholds for gestational diabetes mellitus, are related to the risk of neonatal macrosomia, hypoglycaemia and hyperbilinubinaemia[J]. Diabetologia, 2007, 50(2): 298-306.

[11] The HAPO Study Cooperative Research Group. Hyperglycemia and adverse pregnancy outcomes $[\mathrm{J}]$. N Engl J Med, 2008, 358(19): 1991-2002.

[12] MOOSAZADEH M, ASEMI Z, LANKARANI K B, et al. Family history of diabetes and the risk of gestational diabetes mellitus in Iran: a systematic review and meta-analysis[J]. Diabetes Metab Syndr, 2017, 11 Suppl 1: S99-S104.

[13] KAAJA R. Lipid abnormalities in pre-eclampsia: implications for vascular health $[\mathrm{J}]$. Clin Lipidol, 2011, 6(1): 71-78. 
[14] NELSON T, PEREZ A, ALCARAZ J, et al. Family history of diabetes, acculturation, and the metabolic syndrome among Mexican Americans: proyecto SALSA $[J]$. Metab Syndr Relat Disord, 2007, 5(3): 262-269.

[15] LI H, ISOMAA B, TASKINEN M R, et al. Consequences of a family history of type 1 and type 2 diabetes on the phenotype of patients with type 2 diabetes [J]. Diabetes Care, 2000, 23(5): 589-594.

[16] INOUE K, MATSUMOTO M, MIYOSHI Y, et al. Elevated liver enzymes in women with a family history of diabetes[J/OL]. Diabetes Res Clin Pract, 2008, 79(3): e4-e7.

[17] MOON J H, ROH E, OH T J, et al. Increased risk of metabolic disorders in healthy young adults with family history of diabetes: from the Korea National Health and Nutrition Survey[J]. Diabetol Metab Syndr, 2017, 9(1): 16.

[18 ] LEE Y H, SHIN M H, NAM H S, et al. Effect of family history of diabetes on hemoglobin A1c levels among individuals with and without diabetes: the dong-gu study [J]. Yonsei Med J, 2018, 59(1): 92-100.

[19] ELIRAQI G M, VISTISEN D, LAURITZEN T, et al. Intensive multifactorial treatment modifies the effect of family history of diabetes on glycaemic control in people with Type 2 diabetes: a post hoc analysis of the ADDITION-Denmark randomized controlled trial[ $\mathrm{J}]$. Diabet Med, 2015, 32(8): 1085-1089.

[20] BENNET L, FRANKS P W, ZÖLLER B, et al. Family history of diabetes and its relationship with insulin secretion and insulin sensitivity in Iraqi immigrants and native Swedes: a population-based cohort study $[\mathrm{J}]$. Acta Diabetol, 2018, 55(3): 233-242.

[21] KIM C, NEWTON K M, KNOPP R H. Gestational diabetes and the incidence of type 2 diabetes: a systematic review $[\mathrm{J}]$. Diabetes Care, 2002, 25(10): 1862-1868.

[22] KWAK S H, CHOI S H, JUNG H S, et al. Clinical and genetic risk factors for type 2 diabetes at early or late post partum after gestational diabetes mellitus $[\mathrm{J} / \mathrm{OL}]$. J Clin Endocrinol Metab, 2013, 98(4): E744-E752.

[23] SLADEK R, ROCHELEAU G, RUNG J, et al. A genome-wide association study identifies novel risk loci for type 2 diabetes [J]. Nature, 2007, 445(7130): 881-885.

[24] ZEGGINI E, WEEDON M N, LINDGREN C M, et al. Replication of genome-wide association signals in UK samples reveals risk loci for type 2 diabetes $[\mathrm{J}]$. Science, 2007, 316(5829): 1336-1341.

[25 ] SCOTT L J, MOHLKE K L, BONNYCASTLE L L, et al. A genome-wide association study of type 2 diabetes in Finns detects multiple susceptibility variants $[\mathrm{J}]$. Science, 2007, 316(5829): 1341-1345.

[26] SAXENA R, VOIGHT B F, LYSSENKO V, et al. Genome-wide association analysis identifies loci for type 2 diabetes and triglyceride levels $[\mathrm{J}]$. Science, 2007, 316(5829): 1331-1336.

[27] LAUENBORG J, GRARUP N, DAMM P, et al. Common type 2 diabetes risk gene variants associate with gestational diabetes $[\mathrm{J}]$. J Clin Endocrinol Metab, 2009, 94(1): 145-150.

[28] CHO Y M, KIM T H, LIM S, et al. Type 2 diabetesassociated genetic variants discovered in the recent genome-wide association studies are related to gestational diabetes mellitus in the Korean population $[\mathrm{J}]$. Diabetologia, 2009, 52(2): 253-261.

[29] EGELAND G M, KLUNGSØYR K, ØYEN N, et al. Preconception cardiovascular risk factor differences between gestational hypertension and preeclampsia[J]. Hypertension, 2016, 67(6): 1173-1180.

[30] LEVY A, WIZNITZER A, HOLCBERG G, et al. Family history of diabetes mellitus as an independent risk factor for macrosomia and cesarean delivery $[\mathbf{J}]$. J Matern-Fetal Neonatal Med, 2010, 23(2): 148-152.

[31] SEgal P, HAMilton J K, SERMER M, et al. Maternal obesity and familial history of diabetes have opposing effects on infant birth weight in women with mild glucose intolerance in pregnancy $[\mathrm{J}]$. J MaternFetal Neonatal Med, 2008, 21(1): 73-79.

[32] SCHLEGER F, LINDER K, WALTER L, et al. Family history of diabetes is associated with delayed fetal postprandial brain activity $[\mathrm{J}]$. Front Endocrinol, 2018, 9: 673.

[本文编辑 沈 敏 余 方] 